\title{
ARMADILLO: a Multi-Purpose Cryptographic Primitive Dedicated to Hardware
}

\author{
Stéphane Badel ${ }^{1}$, Nilay Dağtekin ${ }^{1}$, Jorge Nakahara Jr ${ }^{\star 1}$, Khaled Ouafi ${ }^{\star \star}$, Nicolas \\ Reffé $^{2}$, Pouyan Sepehrdad ${ }^{1}$, Petr Sušil ${ }^{1}$, Serge Vaudenay ${ }^{1}$ \\ 1 EPFL, Lausanne, Switzerland \\ 2 Oridao, Montpellier, France \\ \{stephane.badel, nilay.dagtekin, jorge.nakahara, pouyan.sepehrdad, \\ petr.susil, khaled.ouafi, serge.vaudenay\}@epfl.ch, nicolas.reffe@oridao.com
}

\begin{abstract}
This paper describes and analyzes the security of a general-purpose cryptographic function design, with application in RFID tags and sensor networks. Based on these analyzes, we suggest minimum parameter values for the main components of this cryptographic function, called ARMADILLO. With fully serial architecture we obtain that 2923 GE could perform one compression function computation within 176 clock cycles, consuming $44 \mu \mathrm{W}$ at $1 \mathrm{MHz}$ clock frequency. This could either authenticate a peer or hash 48 bits, or encrypt 128 bits on RFID tags. A better tradeoff would use $4030 \mathrm{GE}, 77 \mu \mathrm{W}$ of power and 44 cycles for the same, to hash (resp. encrypt) at a rate of 1.1 Mbps (resp. $2.9 \mathrm{Mbps}$ ). As other tradeoffs are proposed, we show that ARMADILLO offers competitive performances for hashing relative to a fair Figure Of Merit (FOM).
\end{abstract}

\section{Introduction}

Cryptographic hash functions form a fundamental and pervasive cryptographic primitive, for instance, providing data integrity in digital signature schemes, and for message authentication in MACs. In particular, there are very few known hardware-dedicated hash function designs, for instance, Cellhash [6] and Subhash [5]. On the other hand, Bogdanov et al. [2] suggest block-cipher based hash functions for RFID tags using the PRESENT block cipher. Concerning block and stream ciphers, the most prominent developments include PRESENT [1], TEA [22], HIGHT [13], Grain [12], Trivium [4] and KATAN, KTANTAN family [3].

We propose a cryptographic function dedicated to hardware which can be used for several cryptographic purposes. ${ }^{3}$ Such functions rely on data-dependent bit transpositions [16]. Given a bitstring $x=x_{2 k}\|\cdots\| x_{1}$, fixed permutations $\sigma_{0}$ and $\sigma_{1}$ over the set $\{1,2, \ldots, 2 k\}$, a bit string $s$, a bit $b \in\{0,1\}$ and a permutation $\sigma$, define $x_{\sigma_{s}}=x$ when $s$ has length zero, and, $x_{\sigma_{s \mid b}}=x_{\sigma_{s} \circ \sigma_{b}}$, where $x_{\sigma}$ is the bit string $x$ transposed by $\sigma$, that

\footnotetext{
* This work was supported by the National Competence Center in Research on Mobile Information and Communication Systems (NCCR-MICS), a center of the SNF under grant number 5005-67322.

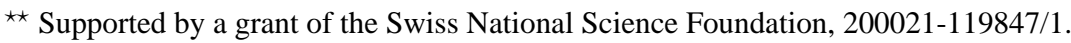

${ }^{3}$ The content of this paper is subject to a pending patent by ORIDAO http://www.oridao.com/.
} 
is, $x_{\sigma}=x_{\sigma(2 k)}\|\cdots\| x_{\sigma(1)}$. The function $(s, x) \mapsto x_{\sigma_{s}}$ is a data-dependent transposition of $x$. The function $s \mapsto \sigma_{s}$ can be seen as a particular case of the general semi-group homomorphism from $\{0,1\}^{*}$ to a group $G$. It was already used in the Zemor-Tillich construction [21] for $G=\mathrm{SL}_{2}$ and in braid group cryptography [10]. We observe that when $\sigma_{0}$ and $\sigma_{1}$ induce an expander graph on the vertex set $v=\{1, \ldots, 2 k\}$, then $(s, x) \mapsto x_{\sigma_{s}}$ has good cryptographic properties.

This paper is organized as follows: Sect. 2 describes a general-purpose cryptographic function called ARMADILLO. In Sect. 3 we analyze ARMADILLO. Sect. 4 contains design criteria for the bit permutation components of ARMADILLO. Sect. 5 suggests parameter vectors. Sect. 6 presents an updated design, called ARMADILLO2. Sect. 7 provides implementation results. Sect. 8 compares hardware implementations of ARMADILLO with other well-known hash functions.

Notations. Throughout this document, \| denotes the concatenation of bitstrings, $\oplus$ denotes the bitwise XOR operation, $\bar{x}$ denotes the bitwise complement of a bitstring $x$; we assume the little-endian numbering of bits, such as $x=x_{2 k}\|\cdots\| x_{1}$.

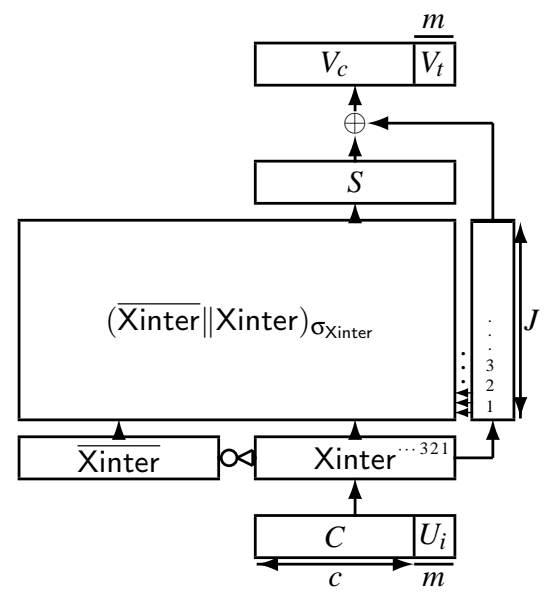

Fig. 1. The ARMADILLO function.

\section{The ARMADILLO Function}

ARMADILLO maps an initial value $C$ and a message block $U_{i}$ to two values

$$
\left(V_{c}, V_{t}\right)=\text { ARMADILLO }\left(C, U_{i}\right)
$$

By definition, $C$ and $V_{c}$ are of $c$ bits, $V_{t}$ as well as each block $U_{i}$ are of $m$ bits, a register Xinter is of $k=c+m$ bits. ARMADILLO is defined by integer parameters $c, m, J=c+m$, and two fixed permutations $\sigma_{0}$ and $\sigma_{1}$ over the set $\{1,2, \ldots, 2 k\}$. $\operatorname{ARMADILLO}(C, U)$ works as follows (see Fig. 1) 
1: set Xinter $=C \| U$;

2: set a $2 k$-bit register $x=\overline{\text { Xinter }} \|$ Xinter;

3: $x$ undergoes a sequence of bit permutations, $\sigma_{0}$ and $\sigma_{1}$, which we denote by $P . P$ maps a bitstring of $k$ bits and a vector $x$ of $2 k$ bits into another vector of $2 k$ bits. Assuming $J=k$, the output of this sequence of $J$ bit permutations is truncated to the rightmost $k$ bits, denoted $S$, by

$$
S=P(\text { Xinter }, x)=\text { tail }_{k}\left((\overline{\text { Xinter }} \| \text { Xinter })_{\sigma_{\text {Xinter }}}\right)
$$

4: set $V_{c} \| V_{t}$ to the value of $S \oplus$ Xinter.

The security is characterized by two parameters $S_{\text {offline }}$ and $S_{\text {online }}$. Concretely, the best offline attack has complexity $2^{S_{\text {offline }}}$, while the best online one, with practical complexity, has success probability $2^{-S_{\text {online }}}$. Typically, we aim at $S_{\text {offline }} \geq 80$ and $S_{\text {online }} \geq 40$. However, we can only upper bound $S_{\text {offline }}$ and $S_{\text {online }}$.

Application I: FIL-MAC. For challenge-response protocols (e.g. for RFID tags [17]), the objective is to have a fixed input-length MAC. Suppose that $C$ is a secret and $U$ is a challenge. The value $V_{t}$ is the response or the authentication tag. We write

$$
V_{t}=\operatorname{AMAC}_{C}(U)
$$

Additionally, the $V_{c}$ output could be used to renew the secret in a synchronized way or to derive an encryption key for a secure messaging session as specified in [17]. The security of challenge-response protocols requires that an adversary cannot extract from the RFID tag enough information that allows it to impersonate the tag with high probability. In this FIL-MAC context, the $C$ parameter can be recovered by exhaustive search with complexity $2^{c}$, where $c=|C|$; so, $S_{\text {offline }} \leq c$. In addition to this, the adversary can try to guess $V_{t}$ online with probability $2^{-m}$, so $S_{\text {online }} \leq m$.

Application II: Hashing and digital signatures. For variable-length input messages hashing, we assume a strengthened Merkle-Damgård [7,15] construction (with padding using length suffix) for ARMADILLO, with $V_{c}$ as chaining variable, $U$ as message block and $V_{c}$ as hash digest. The initial value (IV) can use the fractional part of the square root of 3 truncated to $c$ bits, similar to the values adopted in SHA-2 hash function family [20]. We write

$$
V_{c}=\mathrm{AHASH}_{\mathrm{IV}}(\text { message } \| \text { padding }) .
$$

Generic birthday attacks are expected to find collisions in ARMADILLO with complexity $2^{\frac{c}{2}}$. So, $S_{\text {offline }} \leq \frac{c}{2}$ when collisions are a concern. Preimages and second preimages are expected with probability $2^{-c}$, so, $S_{\text {offline }} \leq c$. Sometimes, free-start collisions or free-start second preimage attacks matter. In this case, we refer to Application II'.

Application III: PRNG and PRF. For pseudorandom generation, we take the first $t$ bits of $V_{c} \| V_{t}$ after at least $r$ iterations. We define

$$
\operatorname{APRF}_{\text {seed }}(x)=\operatorname{head}_{t}\left(\operatorname{AHASH}_{\text {seed }}(x \| \text { cste })\right)
$$


with an input $x$ with length multiple of $m$ and cste a $(r-1) m$-bit constant. A relevant property for this application is indistinguishability. Assuming a secret seed, ARMADILLO could be used as a stream cipher. The keystream is composed of $t$-bit frames where the $i$ th frame is $\operatorname{APRF}_{\text {seed }}(i)$. The index $i$ can be synchronized, or sent in clear in which case we have a self-synchronous stream cipher. In this setting, the output should be indistinguishable from a truly random string when the key is random.

\section{Dedicated Attacks}

Key recovery. Suppose $V_{c} \| V_{t}$ and $U$ are known, and we look for $C$. Since $U$ is known, the tail ${ }_{m}$ (Xinter) are known. Guessing the $\operatorname{tail}_{J-m}(C)$ gives access to the $\operatorname{tail}_{J}(S)$, since $U$ is known. This fact motivates a meet-in-the-middle attack to recover tail $J_{-m}(C)$. Let us split these $J-m$ bits of Xinter into two pieces of sizes $\left\lceil\frac{J-m}{2}\right\rceil$ and $\left\lfloor\frac{J-m}{2}\right\rfloor$. The heading $\left\lceil\frac{J-m}{2}\right\rceil$ bits are used to compute backwards the $P$ permutation from $S$, with $m+\left\lceil\frac{J-m}{2}\right\rceil=$ $\left\lceil\frac{J+m}{2}\right\rceil$ bits known. The tailing $\left\lfloor\frac{J-m}{2}\right\rfloor$ bits of $C$, together with the $m$ bits of $U$ form $m+$ $\left\lfloor\frac{J-m}{2}\right\rfloor=\left\lfloor\frac{J+m}{2}\right\rfloor$ bits of Xinter. The meet-in-the-middle consists in checking consistency of known bits in the middle of the $P$ permutation. We expect to find a solution with complexity $O\left(2^{\left\lceil\frac{J+m}{2}\right\rceil}\right)$ and a single $U$. Thus, $S_{\text {offline }} \leq\left\lceil\frac{J+m}{2}\right\rceil$ in Application I, II and III.

Free-start collision. We look for a triplet $\left(C, U, U^{\prime}\right)$ that causes a collision, that is, $\mathrm{AHASH}_{C}(U)=\mathrm{AHASH}_{C}\left(U^{\prime}\right)$. For this, we look for $\left(C, U, U^{\prime}\right)$ such that

$$
P(U, \bar{C}\|\bar{U}\| C \| U) \approx P\left(U^{\prime}, \bar{C}\left\|\overline{U^{\prime}}\right\| C \| U^{\prime}\right)
$$

with $\approx$ meaning that the Hamming weight of the difference is some low value $w$. Then, we hope that the next $P$ permutation will move all $w$ different bits outside the window of the $c+m$ bits which are kept in $S$. Since the probability for a vector to have weight $w$ is $\left(\begin{array}{c}2 c+2 m \\ w\end{array}\right) 2^{-2 c-2 m}$, the number of solutions we get is $\left(\begin{array}{c}2 c+2 m \\ w\end{array}\right) 2^{-c}$ on average. The probability that a solution leads to a collision is the probability that $w$ difference bits are moved outside a window of $c$ bits. Finally, the expected number of collisions we can get is $\left(\begin{array}{c}c+m \\ w\end{array}\right) 2^{-c}$. We can now fix $w=w_{\text {opt }}$ such that $\left(\begin{array}{c}c+m \\ w_{\mathrm{opt}}\end{array}\right) \geq 2^{c}$ so that we can find one solution with complexity $2^{w_{\text {opt }}}$. To implement the attack, for all $U$ and $U^{\prime}$ we enumerate all $C$ 's such that $P(U, \bar{C}\|\bar{U}\| C \| U) \approx P\left(U^{\prime}, \bar{C}\left\|\overline{U^{\prime}}\right\| C \| U^{\prime}\right)$. The complexity is $2^{2 m}+\left(\begin{array}{c}2 k \\ w_{\mathrm{opt}}\end{array}\right) 2^{-c}$ which is dominated by $2^{2 m}$. So, $S_{\text {offline }} \leq 2 m$ in Application II'.

A distinguisher. Assuming that the $J$ iterations in the $P$ permutation output a random $2 k$-bit vector of Hamming weight $k$, we have $\left(\begin{array}{c}2 k \\ k\end{array}\right)$ possible vectors. By extracting a window of $t$ bits we do not have a uniformly distributed string. Indeed, any possible string of weight $w$ has a probability of $p(w)=\left(\begin{array}{c}2 k-t \\ k-w\end{array}\right) /\left(\begin{array}{c}2 k \\ k\end{array}\right)$. There exists a distinguisher to tell whether a $t$-bit window comes from a random output from $P$ or a truly random string, with advantage

$$
\frac{1}{2} \sum_{w=0}^{t}\left(\begin{array}{l}
t \\
w
\end{array}\right)\left|\frac{\left(\begin{array}{c}
2 k-t \\
k-w
\end{array}\right)}{\left(\begin{array}{c}
2 k \\
k
\end{array}\right)}-\frac{1}{2^{t}}\right|
$$


For $t=k=160$, this is 0.1658 . Here, the distinguisher recognizes $P$ when the Hamming weight $w$ is in the interval $[75, \ldots, 85]$, and a random string otherwise.

The final XOR hides this bias a bit but we can wonder by how much exactly. Assume that we hash a message of $r$ blocks. The final output is the XOR of the initial value together with $r$ outputs from $P$. Assuming that the initial value is known and that the $P$ outputs are random and independent, we can compute the distribution of the final hash by convolution. Indeed, the probability that it is a given string $x$ is $p_{r}(x)$ such that

$$
p_{r}(x)=\sum_{x_{1} \oplus \cdots \oplus x_{r}=x} p\left(\mathrm{wt}\left(x_{1}\right)\right) \cdots p\left(\mathrm{wt}\left(x_{r}\right)\right)
$$

Let us define the spectrum $\hat{p}_{r}(\mu)$ by $\hat{p}_{r}(\mu)=\sum_{x}(-1)^{\mu \cdot x} p_{r}(x)$. We have $\hat{p}_{r}(\mu)=\left(\hat{p}_{1}(\mu)\right)^{r}$. We can now compute

$$
\hat{p}_{1}(\mu)=\sum_{i=0}^{\mathrm{wt}(\mu)} \sum_{j=0}^{t-\mathrm{wt}(\mu)}\left(\begin{array}{c}
\mathrm{wt}(\mu) \\
i
\end{array}\right)\left(\begin{array}{c}
t-\mathrm{wt}(\mu) \\
j
\end{array}\right)(-1)^{i} p(i+j)
$$

It only depends on $\operatorname{wt}(\mu)$ so we write $\hat{p}_{1}(w t(\mu))$. Since $\sum_{\mu} \hat{p}_{r}(\mu)^{2}=2^{t} \sum_{x} p_{r}(x)^{2}$ we deduce that the Squared Euclidean Imbalance (SEI) of the difference of the hash of $r$ blocks with the initial value is

$$
\mathrm{SEl}_{r}=2^{t} \sum_{x}\left(p_{r}(x)-2^{-t}\right)^{2}=\sum_{\mu \neq 0}\left(\hat{p}_{r}(\mu)\right)^{2}=\sum_{w=1}^{t}\left(\begin{array}{c}
t \\
w
\end{array}\right)\left(\hat{p}_{1}(w)\right)^{2 r}
$$

We have $S_{\text {offline }} \leq-\log _{2} \mathrm{SEI}_{r}$, where $r$ is the minimal number of blocks which are processed in Application III. The SEI expresses as

$$
\mathrm{SEI}_{r}=\sum_{w=1}^{t}\left(\begin{array}{l}
t \\
w
\end{array}\right)\left(\sum_{i=0}^{w}\left(\begin{array}{c}
w \\
i
\end{array}\right) \sum_{j=0}^{t-w}\left(\begin{array}{c}
t-w \\
j
\end{array}\right)(-1)^{i} \frac{\left(\begin{array}{c}
2 k-t \\
k-i-j
\end{array}\right)}{\left(\begin{array}{c}
2 k \\
k
\end{array}\right)}\right)^{2 r}
$$

As an example, we computed $\mathrm{SEl}_{r}$ for four selections of $t=k$.

\begin{tabular}{r|c|c|c|c}
\multicolumn{3}{|c}{$t=k=128$} & $t=k=160 \mid t=k=200$ & $t=k=275$ \\
\hline$r$ & \multicolumn{4}{|c}{$\mathrm{SEI}_{r}$} \\
\hline 1 & $2^{-2.70}$ & $2^{-2.70}$ & $2^{-2.70}$ & $2^{-2.70}$ \\
2 & $2^{-18.99}$ & $2^{-19.63}$ & $2^{-20.28}$ & $2^{-21.20}$ \\
3 & $2^{-34.98}$ & $2^{-36.27}$ & $2^{-37.56}$ & $2^{-39.40}$ \\
4 & $2^{-50.96}$ & $2^{-52.90}$ & $2^{-54.81}$ & $2^{-57.60}$ \\
5 & $2^{-66.95}$ & $2^{-69.54}$ & $2^{-72.12}$ & $2^{-75.81}$ \\
6 & $2^{-82.94}$ & $2^{-86.17}$ & $2^{-89.40}$ & $2^{-94.01}$ \\
7 & $2^{-98.93}$ & $2^{-102.81}$ & $2^{-106.68}$ & $2^{-112.21}$
\end{tabular}

Given $k$ and $c$, we look for $r$ and $t$ such that $\mathrm{SEI}_{r}<2^{-c}$ and $r / t$ is minimal.

\section{Permutation-Dependent Attacks}

In this section we present security criteria for the $\sigma_{0}$ and $\sigma_{1}$ permutations. 
Another distinguisher. Consider a set $I$ of indices from $V=\{1, \ldots, 2 k\}$. Let $\operatorname{swap}_{I}(\sigma)=$ $\#\{i \in I ; \sigma(i) \notin I\}$ and $\mathrm{wt}_{I}(x)=\sum_{i \in I} x_{i}$. We assume that $s_{b}=\operatorname{swap}_{I}\left(\sigma_{b}\right)$ is low for $b=0$ and $b=1$ to see how much the low diffusion between inside and outside $I$ would lead to a distinguisher on $P(s, \cdot)$ with a random $s$ of $J$ bits. In the worst case we can assume that all indices in $I$ are in the same half of $x$ so that the distinguisher can choose the input on $P$ with a very biased $\mathrm{wt}_{I}(x)$.

A permutation $\sigma_{b}$ keeps $\# I-s_{b}$ of the bits inside $I$ and introduce $s_{b}$ bits from outside $I$. Assuming that all bits inside and outside $I$ are randomly permuted, we have the approximation

$$
E\left(\mathrm{wt}_{I}\left(x_{\sigma_{b}}\right)\right) \approx\left(\# I-s_{b}\right) \frac{E\left(\mathrm{wt}_{I}(x)\right)}{\# I}+s_{b} \frac{k-E\left(\mathrm{wt}_{I}(x)\right)}{2 k-\# I} .
$$

Thus,

$$
E\left(\mathrm{wt}_{I}\left(x_{\sigma_{b}}\right)\right)-\frac{\# I}{2} \approx\left(1-\frac{s_{b}}{\# I}-\frac{s_{b}}{2 k-\# I}\right)\left(E\left(\mathrm{wt}_{I}(x)\right)-\frac{\# I}{2}\right) .
$$

On average over the control bits, we have

$$
\frac{E\left(\mathrm{wt}_{I}(P(s, x))\right)-\frac{\# I}{2}}{E\left(\mathrm{wt}_{I}(x)\right)-\frac{\# I}{2}} \approx\left(1-\frac{s_{0}+s_{1}}{2} \times \frac{2 k}{\# I(2 k-\# I)}\right)^{J} .
$$

The best strategy for the distinguisher consists of having either $\mathrm{wt}_{I}(x)=0 \mathrm{orwt}_{I}(x)=$ $\# I$. In both cases we have

$$
\left|E\left(\mathrm{wt}_{I}(P(s, x))\right)-\frac{\# I}{2}\right| \approx \frac{\# I}{2}\left(1-\frac{s_{0}+s_{1}}{2} \times \frac{2 k}{\# I(2 k-\# I)}\right)^{J} .
$$

The number of samples to significantly observe this bias is

$$
T=\left(1-\frac{s_{0}+s_{1}}{2} \times \frac{2 k}{\# I(2 k-\# I)}\right)^{-2 J} .
$$

So, $S_{\text {offline }} \leq \log _{2} T$. This expression relates to the theory of expander graphs. We provide below a sufficient condition which can be easily checked.

To compute the minimal value of $\frac{s_{0}+s_{1}}{2 \# I}$ over all $I$ we observe that if $P_{\sigma_{b}}$ is the matrix of permutation $\sigma_{b}$ and if $x_{I}$ is the $0-1$ vector whose coordinate of index in $I$ are the ones set to 1 , then

$$
\frac{s_{0}+s_{1}}{2 \# I}=1-\frac{x_{I} \cdot\left(\frac{P_{\sigma_{0}}+P_{\sigma_{1}}}{2} x_{I}\right)}{x_{I} \cdot x_{I}} .
$$

Let $u$ be the vector with all coordinates set to 1 . Clearly, the hyperplane $u^{\perp}$ orthogonal to $u$ is stable by the matrix $M_{0}=\frac{1}{2}\left(P_{\sigma_{0}}+P_{\sigma_{1}}\right)$. Let $M=\frac{1}{2}\left(M_{0}+M_{0}^{t}\right)$, where the superscript indicates the transpose matrix. We can easily see that $M u=u$. Furthermore, we notice that $M x=\lambda x$ with $x \neq 0$ implies $|\lambda| \leq 1$. Let $\lambda$ be the second largest eigenvalue of $M$, or equivalently the largest eigenvalue of operator $M$ restricted to $u^{\perp}$. Note that $\lambda$ can be $\lambda=1$ if the eigenvalue 1 has multiplicity higher than one. We can easily prove that $|\lambda|=1$ and $M x=\lambda x$ with $x \neq 0$ implies that $x_{i}$ is constant for all $i \in I$, for all connected 
components $I$ for the relation $i \sim j \Longleftrightarrow \exists s \quad \sigma_{s_{|s|}} \circ \cdots \circ \sigma_{s_{2}} \circ \sigma_{s_{1}}(i)=j$. Hence, the only sets $I$ which are stable by $\sigma_{0}$ and $\sigma_{1}$ at the same time are the empty one and the complete set if and only if eigenvalue 1 has multiplicity one. So, having $\lambda<1$ is already a reasonable criterion but we can have a more precise one. We know that for any vector $x$ orthogonal to $u$ we have $\frac{x \cdot\left(M_{0} x\right)}{x \cdot x} \leq \lambda$ with equality when $x$ is an eigenvector for $\lambda$. Thus,

$$
\frac{x \cdot\left(M_{0} x\right)}{x \cdot x} \leq \frac{(1-\lambda) \frac{(x \cdot u)^{2}}{u \cdot u}+\lambda(x \cdot x)}{x \cdot x}
$$

for any $x \neq 0$. For $x=x_{I}$ we obtain

$$
\frac{x_{I} \cdot\left(M_{0} x_{I}\right)}{x_{I} \cdot x_{I}} \leq(1-\lambda) \frac{\# I}{2 k}+\lambda .
$$

From (2), $\frac{s_{0}+s_{1}}{2 \# I}=1-\frac{x_{I} \cdot\left(M_{0} \cdot x_{I}\right)}{x_{I} \cdot x_{I}} \geq 1-(1-\lambda) \frac{\# I}{2 k}+\lambda=(1-\lambda)\left(1-\frac{\# I}{2 k}\right)$. Going back to the complexity (1) of our distinguisher we have $T \geq \lambda^{-2 J}$. Hence, by having $\lambda \leq 2^{-\frac{S_{\text {offline }}}{2 J}}$ for an offline complexity $2^{S_{\text {offline }}}$, we make sure that the distinguisher has complexity $T \geq 2^{S_{\text {offline }}}$. To conclude, if $\lambda$ is the second largest eigenvalue of $M=\frac{1}{4}\left(P_{\sigma_{0}}+P_{\sigma_{0}}^{t}+\right.$ $\left.P_{\sigma_{1}}+P_{\sigma_{1}}^{t}\right)$ then we have an attack of complexity $\lambda^{-2 J}$. So, $S_{\text {offline }} \leq-2 J \log _{2} \lambda$.

Yet another distinguisher. We define the vector $x$ of dimension $k$ such that the $i$ th coordinate of $x$ is the probability that $x_{i}$ is set to 1 . If $x$ is fixed, we can consider that $x$ is equal to $x$ by abuse of notation. If $y=x_{\sigma}$, we have that $y$ is obtained by multiplying a permutation matrix $P_{\sigma}$ by $x$. We have $\left(P_{\sigma}\right)_{j, i}=1$ if and only if $j=\sigma(i)$. Clearly, for $y=x_{\sigma_{b}}$ we can write

$$
\begin{aligned}
y & =\left((1-b) P_{\sigma_{0}}+b P_{\sigma_{1}}\right) \times x \\
& =\left(\frac{1}{2}\left(P_{\sigma_{0}}+P_{\sigma_{1}}\right)+\frac{(-1)^{b}}{2}\left(P_{\sigma_{0}}-P_{\sigma_{1}}\right)\right) \times x
\end{aligned}
$$

We let $M_{0}=\frac{1}{2} P_{\sigma_{0}}+\frac{1}{2} P_{\sigma_{1}}$ and $M_{1}=\frac{1}{2} P_{\sigma_{0}}-\frac{1}{2} P_{\sigma_{1}}$. We have

$$
\prod_{i=1}^{J}\left(M_{0}+(-1)^{s_{i}} M_{1}\right)=\sum_{a_{1}=0}^{1} \cdots \sum_{a_{k}=0}^{1}(-1)^{a_{1} s_{1}+\cdots+a_{k} s_{k}} M_{a}=\hat{M}_{s}
$$

where $M_{a}=M_{a_{1}} \times \cdots \times M_{a_{k}}$. So, the vector of $y=P(s, x)$ is $y=\hat{M}_{s} x$. The average $\left\langle\hat{M}_{s}\right\rangle$ of $\hat{M}_{s}$ over all $s_{1}, \ldots, s_{k}$ is $M_{0}^{k}$. We define a square matrix $F$ in which all terms are equal to $\frac{1}{k}$. Clearly, if $s$ is a uniformly distributed $J$-bit random string, the probability vector of $P(s, x)$ is $M_{0}^{J} \times x$. Since $M_{0}$ is a bi-stochastic matrix, we have $M_{0} \times F=F \times M_{0}=F$. Similarly, we have $M_{1} \times F=F \times M_{1}=0$. We easily deduce that $\left(M_{0}-F\right)^{J}=M_{0}^{J}-F$. Let $\theta$ be the second largest eigenvalue of $M_{0}^{t} M_{0}$, or equivalently, the largest eigenvalue of $M_{0}^{t} M_{0}-F$. For any vector $x$ such that $\sum x_{i}=w$ and $0 \leq x_{i} \leq 1$, we have $\left\|M_{0}^{J} x-w u\right\|_{2}^{2}=\left\|\left(M_{0}-F\right)^{J} x\right\|_{2}^{2} \leq w \theta^{J}$, where $u=(1, \ldots, 1)$. So, the cumulated squared Euclidean imbalance of each component of $M_{0}^{J} x$ is bounded by $2 k \theta^{J}$. Thus, the complexity is $\frac{1}{2 k \theta^{J}}$, and $S_{\text {offline }} \leq-J \log _{2} \theta-\log _{2} 2 k$. The average $\left\langle\hat{M}_{s}\left(\begin{array}{l}\bar{s} \\ s\end{array}\right)\right\rangle$ of the image of 
Xinter $=s$ is

$$
\sum_{a} M_{a}\left\langle(-1)^{a \cdot s}\left(\begin{array}{l}
\bar{s} \\
s
\end{array}\right)\right\rangle .
$$

For $a=0$ the average is $\left(\frac{1}{2} \cdots \frac{1}{2}\right)$. For $a$ of weight at least 2, the average is zero. For $a$ of weight 1 , e.g. $a=(1,0, \ldots, 0)$ the average is $\left(-\frac{1}{2}, 0, \ldots, 0, \frac{1}{2}, 0, \ldots, 0\right)$. We let $e_{i}$ be the vector with coordinate 1 in its $i$ th position and 0 elsewhere. We have

$$
\left\langle\hat{M}_{s}\left(\begin{array}{c}
\bar{s} \\
s
\end{array}\right)\right\rangle=\left(\begin{array}{c}
\frac{1}{2} \\
\vdots \\
\frac{1}{2}
\end{array}\right)+\frac{1}{2} \sum_{i=1}^{k} M_{0}^{i-1} M_{1} M_{0}^{k-i}\left(-e_{i}+e_{k+i}\right)
$$

Let

$$
b=\frac{1}{2} \sum_{i=1}^{k} M_{0}^{i-1} M_{1} M_{0}^{k-i}\left(-e_{i}+e_{k+i}\right) .
$$

The complexity is $\frac{1}{2\|b\|_{2}^{2}}$, so, we have $S_{\text {offline }} \leq-2 \log _{2}\|b\|_{2}-1$.

The parity of $P$. Let $\varepsilon_{i}$ be the parity of $\sigma_{i}$. The $x \mapsto P(s, x)$ is a permutation whose parity is $\varepsilon_{0}^{|s|-\mathrm{wt}(s)} \varepsilon_{1}^{\mathrm{wt}(s)}$. If $\varepsilon_{0} \neq \varepsilon_{1}$, an adversary with black-box access to $x \mapsto P(s, x)$ and knowing $|s|$ can thus easily deduce wt $(s)$. We thus, recommend that $\varepsilon_{0}=\varepsilon_{1}$.

\section{Parameter Vectors}

Here we suggest sets of parameters for four different applications, based on our analyzes. In all cases, we require $J=c+m$ and also that $\sigma_{0}$ and $\sigma_{1}$ have the same parity.

I: in a challenge-response application: $S_{\text {offline }} \leq \min \left(c, \frac{J+m}{2}\right)$ and $S_{\text {online }} \leq m$

II: in a collision-resistance context: $S_{\text {offline }} \leq \frac{c}{2}$

II': in a free-start collision context: $S_{\text {offline }} \leq \min \left(\frac{c}{2}, 2 m\right)$

III: $S_{\text {offline }} \leq-\log _{2} \mathrm{SEl}_{r}$. If $\lambda$ is the second largest eigenvalue of $M=\frac{1}{4}\left(P_{\sigma_{0}}+P_{\sigma_{0}}^{t}+\right.$ $\left.P_{\sigma_{1}}+P_{\sigma_{1}}^{t}\right)$ then $S_{\text {offline }} \leq-2 J \log _{2} \lambda$. For $\sigma_{0}$ and $\sigma_{1}$, the second largest eigenvalue of $M_{0}^{t} M_{0}$, called $\theta, S_{\text {offline }} \leq-J \log _{2} \theta-\log _{2} k-1$. The bias $b$ in (4) shall satisfy $S_{\text {offline }} \leq-2 \log _{2}\|b\|_{2}-1$. Moreover, $S_{\text {offline }} \leq \frac{J+m}{2}$.

To match the ideal security, we need these bounds to yield $S_{\text {offline }} \leq c$ and $S_{\text {online }} \leq m$ for Application I, $S_{\text {offline }} \leq \frac{c}{2}$ for Application II and II', and $S_{\text {offline }} \leq c$ for Application III. So, we take $J=c+m, m \geq \frac{c}{2} ; r$ and $t$ such that $\mathrm{SEI}_{r} \leq 2^{-c} ; \sigma_{0}$ and $\sigma_{1}$ such that $-\log _{2} \lambda \geq \frac{c}{2(c+m)},-\log _{2} \theta \geq \frac{c+\log 2 k}{c+m},-\log _{2}\|b\|_{2} \geq \frac{c+1}{2}$, and $\varepsilon_{0}=\varepsilon_{1}$. Our recommendations for the parameter values of ARMADILLO are given in Table 1. Note that $c$ is the key length for Applications I and III and also the digest length for Application II. 
Table 1. Parameter vectors.

\begin{tabular}{c|cccccc}
\hline Vector & $k$ & $J$ & $c$ & $m$ & $r$ & $t$ \\
\hline $\mathrm{A}$ & 128 & 128 & 80 & 48 & 6 & 128 \\
$\mathrm{~B}$ & 192 & 192 & 128 & 64 & 9 & 192 \\
$\mathrm{C}$ & 240 & 240 & 160 & 80 & 10 & 240 \\
$\mathrm{D}$ & 288 & 288 & 192 & 96 & 12 & 288 \\
$\mathrm{E}$ & 384 & 384 & 256 & 128 & 15 & 384 \\
\hline
\end{tabular}

\section{ARMADILLO2}

Ever since the first version of ARMADILLO, we have developed an updated design, called ARMADILLO2, that is even more robust than the version presented in Fig. 1. In fact, ARMADILLO2 brings in a new compression function, called $Q$, which is not only more compact in hardware than $P$, but also addresses security concerns brought about during the continuous analyzes of ARMADILLO. For these reasons, ARMADILLO2 is our preferred design choice. Due to space limitations, further details about the security analysis of ARMADILLO2 are omitted. ARMADILLO2 is defined by

$$
\left(V_{c}, V_{t}\right)=\operatorname{ARMADILLO} 2(C, U)=Q(X, C \| U) \oplus X, \text { where } X=Q(U, C \| U) .
$$

We call the new permutation $Q$, instead of $P$ as in Fig. 1, to avoid confusion. The main novelties are:

- there is no complementation of the $k$-bit input Xinter $=C \| U$ anymore; as a consequence, the $\sigma_{i}$ permutations (and therefore $Q$ ) now operate on $k$-bit data $C \| U$, instead of $\bar{C}\|\bar{U}\| C \| U$, leading to a more compact design;

- a new permutation $Q$ which interleaves $\sigma_{i}$ 's, $i \in\{0,1\}$, with an xor using the $k$-bit constant bitstring $\gamma=1010 \cdots 10 ; Q$ is defined recursively as $Q(s \| b, X)=$ $Q\left(s, X_{\sigma_{b}} \oplus \gamma\right)$ and $Q(\emptyset, X)=X$, for $b \in\{0,1\}$ and bitstrings $s$ and $X$;

- the outermost $Q$ is controlled by a data-dependent value, $X=Q(U, C \| U)$, in contrast to simply $C \| U$ in Fig. 1;

In the new structure of $Q$, the output bias disappears and we can take $r=1$ and $t=k$.

\section{Hardware Implementation and Performance}

There exist different demands on the implementation and the optimization meanings for various application scenarios. In this context, the scalability of ARMADILLO allows to deploy the implementation in a very wide realm of area and speed parameters, which constitutes the most essential trade-off in electronics circuits. The implementation of the $P$ function, using the building block, is depicted in Fig. 2(b). It accepts an input vector of $2 k$ bits and a key of $J$ bits. It consists of a variable number $N$ of permutation stages, all identical, and each stage essentially requires $2 k$ multiplexers (Fig. 2(a)). One register of 
$2 k$ bits is needed to hold the input and/or intermediate data, as well as one $J$-bit register to hold the permutation key. At each cycle, these registers are either loaded with new data/key or fed back the output data/key for a new permutation round, depending on the state of the load signal. The number $N$ of permutations executed in each cycle can be adjusted, the only restriction being that $J$ be an integer multiple of $N$. The output data is the $2 k$ bits vector resulting from the permutation round, and the output key is the $J-N$ bits remaining to be processed. This building block can be flexibly assembled into a

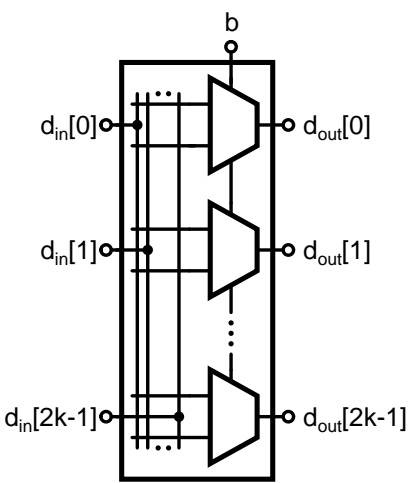

(a)

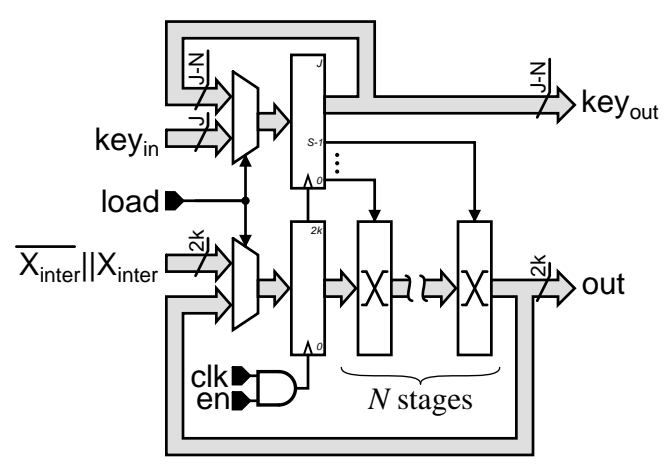

(b)

Fig. 2. Hardware implementation of the ARMADILLO function. (a) one permutation stage. (b) $P$ function building block.

$T$-stage pipeline, where each stage performs a number $R=J /(N \cdot T)$ of permutation rounds (building blocks) before passing the results to the next stage and accepting new input from the previous stage. In that case, the throughput is $1 / R$ items per cycle and the latency is $J / N$ cycles, the parameters being linked by the equality $R \cdot N \cdot T=J$. The latency / throughput / cost trade-off can be adjusted, the two extreme cases being $R=1$ (fully pipelined, resulting in a throughput of 1 item per cycle) and $T=1$ (fully serial, resulting in a throughput of $S / J$ items per cycle). Obviously, the more pipeline stages, the more hardware replication and therefore the higher the cost in area and power. To construct the complete hash function of Fig. 1, we essentially need to add a state machine (which is little more than a counter) around the permutation function block, and the final XOR operation.

Metrics for evaluating performance In order to compare different cryptographic functions, several metrics can be taken into account. The security is of course the primary concern. The silicon area, the throughput, the latency and the power dissipation are other metrics of interest, and can be traded-off for one another. For example, the power dissipation is nearly proportional to the clock frequency in any CMOS circuit, therefore, power can be reduced by decreasing the clock frequency and thus at the expense of throughput. Conversely, throughput can be increased by running at a faster clock 
frequency, up to a maximum clock frequency which is process- and implementationdependent. Another example is serialization, where an operation is broken into several steps executed in series, allowing to reuse the same hardware, but again at the cost of a longer execution time. Through serialization, throughput and latency can be traded-off for area, down to a point where operations can not be broken into smaller operations anymore and we have reached a minimum area. Given this large design space, comparing the relative merit of different cryptographic functions is a challenging task.

The approach taken in [2] (and numerous other publications) includes comparing the area of synthesized circuits as reported in the literature or estimated by the authors in gate-equivalent (GE). It is notable though that the GE unit of measure, while being convenient because it is process-independent, is very coarse. For example, does the reported area after synthesis include the space needed for wiring? Typically, the utilization of a routed circuit can be in the range of $50 \%-80 \%$, and is especially critical when using a limited number of metal layers for routing. A synthesis tool may report an estimated routing area, but in all cases it may vary to a large extent after physical implementation. Consider also that one design may have scan chains inserted while another may not, which may increase the register area by as much as $20-30 \%$ and require extra interconnections. Furthermore, different standard cells may be of varying area efficiency; as an illustration of this fact, a comparison of gate-equivalent figures from different standard-cell libraries can produce different results with a ratio up to $\frac{2}{3}$. For instance, a simple 2-input multiplexer can lead to 2.67 GE or 1.67 GE from one library to the other. Taking into account all these factors, it is clear that such a comparison can have a large margin of error, unless the circuits being compared have been implemented in the exact same conditions.

Besides comparing areas, the authors of [2] also use a metric called efficiency, which is defined as the ratio of the throughput (measured at a fixed clock frequency) over the area. It may seem at first sight that such a metric provides a more general measure of quality, since it may be fair to give up some area for a higher throughput, however it is flawed in that it does not consider the possibility of trading off throughput for power. Indeed, according to this metric, two designs A and B would be deemed of equal value if, for example, A's throughput and area were twice B's throughput and area, respectively. However, if B's power dissipation is half that of A at the same clock frequency, then by doubling B's operating frequency, its throughput can be made equal to that of A while consuming the same power and still occupying a smaller area. Clearly then, B should be recognized as superior to $\mathrm{A}$, which can be captured by dividing the metric by the power dissipation, thus making it independent of the power/throughput trade-off. However, this does not come without its own problems, since the power dissipation is an extremely volatile quantity. Being subject to the same error factors as the area as described above, it also depends heavily on the process technology, the supply voltage, and the parasitic capacitances due to the interconnections. Furthermore, it can vary largely depending on the method used to measure it (i.e. gate-level statistical or vector-based simulation, or SPICE simulation). As if this were not enough, different standard-cell libraries also exhibit various power/area/speed trade-offs, for example, a circuit implemented with a high-density library is likely to result in a lower power figure than the same circuit implemented with a general-purpose library, for a similar gate count. 
Nevertheless, a fairer figure of merit would need to include the influence of power dissipation. In order to keep process-independent metrics, we can assume that the power is proportional to the gate count. ${ }^{4}$ This is reasonable since the dynamic power in CMOS circuits is proportional to the total switched capacitance, which correlates to the area. We propose therefore to use a figure of merit defined as $\mathrm{FOM}=$ throughput $/ \mathrm{GE}^{2}$. In practice, this is a coarse approximation, since it does not take into account switching activity or the influence of wire load; it is nevertheless fairer than not including power dissipation at all, since it tends to favor designs with smaller area (at equal throughput) which are very likely to dissipate less power.

Synthesis Results Table 2 presents the results of synthesis for the hash function described above in a $0.18 \mu \mathrm{m}$ CMOS process using a commercial standard-cell library, with the parameters given in Sect. 5. Synthesis was performed with Synopsys Design Compiler in topographical mode, in order to obtain accurate wire loads. The power consumption was evaluated with Synopsys Primetime-PX using gate-level vector-based analysis.

In RFID applications, the latency is constrained by the communication protocols (though the constraint is relatively easily satisfiable) but a high throughput is not necessary, designating a fully serial implementation as the ideal candidate. Therefore $T$ is set to $T=1$. The number $N$ of permutations per clock cycle in the permutation function is set to $N=1$, which is favorable to smaller area and power consumption for the tight power budget associated with RFID applications. The clock frequency is set to $1 \mathrm{MHz}$, which is a representative value for the target application.

In hash mode we hash $m$ bits per compression. In encryption mode we encrypt $t / r$ bits per compression. The throughput values given in Table 2 correspond to hash mode.

Our goal for selecting $T=1$ and $N=1$ was to minimize the hardware. The area in the proposed implementation is roughly proportional to

$$
\left(k_{\mathrm{reg}} *(2 k+J)+k_{\log } *(2 k(N+1)+J)\right) T
$$

for some constants $k_{\text {reg }}$ and $k_{\log }$.

To maximize the FOM with $T$ given, we can show that we should in theory pick

$$
N=\left(\frac{k_{\mathrm{reg}}}{k_{\mathrm{log}}}+1\right)\left(1+\frac{J}{2 k}\right)
$$

For $k_{\text {reg }} \approx 2 k_{\log }$ and $J=k$, this is $N=4.5$. In practice, the best choice is to take $T=1$ and $N=4$ for ARMADILLO2 in context A, for which we would get an area of $4030 \mathrm{GE}$, $77 \mu \mathrm{W}$, and a latency of 44 cycles (1.09 Mbps for hashing or 2.9 Mbps for encryption).

\section{Comparison}

Table 3 shows a comparison of hardware implementations of ARMADILLO in the hash function setting, relative to other hash functions such as MD4, MD5, SHA-1, SHA-

\footnotetext{
${ }^{4}$ In the same spirit as the GE unit of measure, a more interesting metric would be to divide the power by $C_{u n i t} \cdot V_{D D}^{2}$, where $C_{\text {unit }}$ is the input capacitance of an inverter. However, this is not applicable to compare other published implementations since these quantities are usually unknown
} 
Table 2. Synthesis results at $1 \mathrm{MHz}$.

\begin{tabular}{c|cccc|cccc}
\hline \multirow{2}{*}{ Algorithm } & \multicolumn{9}{|c|}{$\begin{array}{c}\mathrm{N}=1 \\
\text { Area }\end{array}$} & $\begin{array}{c}\text { Power } \\
\text { Throughput Latency } \\
\end{array}$ & $(\mathrm{GE})$ & $(\mu \mathrm{W})$ & $\begin{array}{c}\text { Area } \\
(\mathrm{kbps})\end{array}$ & $\begin{array}{c}\text { Power } \\
(\mathrm{cycles})\end{array}$ & $\begin{array}{c}\mathrm{N}=4 \\
(\mathrm{GE})\end{array}$ & $(\mu \mathrm{W})$ & $(\mathrm{kbps})$ & $\begin{array}{c}\text { Latency } \\
(\mathrm{cycles})\end{array}$ \\
\hline ARMADILLO-A & 3972 & 69 & 375 & 128 & 5770 & 133 & 1500 & 32 \\
ARMADILLO-B & 6598 & 117 & 333 & 192 & 9709 & 237 & 1333 & 48 \\
ARMADILLO-C & 8231 & 146 & 333 & 240 & 12217 & 300 & 1333 & 60 \\
ARMADILLO-D & 8650 & 177 & 333 & 288 & 14641 & 368 & 1333 & 72 \\
ARMADILLO-E & 13344 & 228 & 333 & 384 & 19669 & 513 & 1333 & 96 \\
\hline ARMADILLO2-A & 2923 & 44 & 272 & 176 & 4030 & 77 & 1090 & 44 \\
ARMADILLO2-B & 4353 & 65 & 250 & 256 & 6025 & 118 & 1000 & 64 \\
ARMADILLO2-C & 5406 & 83 & 250 & 320 & 7492 & 158 & 1000 & 80 \\
ARMADILLO2-D & 6554 & 102 & 250 & 384 & 8999 & 183 & 1000 & 96 \\
ARMADILLO2-E & 8653 & 137 & 250 & 512 & 11914 & 251 & 1000 & 128 \\
\hline
\end{tabular}

256, and MAME according to [2]. We computed the throughput in kbps at a clock rate of $100 \mathrm{kHz}$. We added the best FOM results for KATAN and KTANTAN with 64-bit blocks from [3]. Algorithms are categorized in terms of security by taking into account the digest size. In each category, we listed the algorithms by decreasing order of merit. To estimate the FOM we assumed that the power was proportional to the area. So, it is the speed divided by the square of the area. These figures show that different versions of ARMADILLO2 provide clear advantage for hashing, either in terms of area, or of throughput, or of overall merit.

Table 3. Implementation comparison for hash functions with throughput at $100 \mathrm{kHz}$.

\begin{tabular}{cccccccc}
\hline Algorithm & $\begin{array}{c}\text { Digest } \\
\text { (bits) }\end{array}$ & $\begin{array}{c}\text { Block } \\
\text { (bits) }\end{array}$ & $\begin{array}{c}\text { Area } \\
(\mathrm{GE})\end{array}$ & $\begin{array}{c}\text { Time } \\
\text { (cycles/block) }\end{array}$ & $\begin{array}{c}\text { Throughput } \\
(\mathrm{kb} / \mathrm{s})\end{array}$ & $\begin{array}{c}\text { Logic } \\
(\mu \mathrm{m})\end{array}$ & $\begin{array}{c}\text { FOM } \\
\text { (nanobit/cycle.GE }{ }^{2} \text { ) }\end{array}$ \\
\hline \hline ARMADILLO2-A & 80 & 48 & 4030 & 44 & 109 & 0.18 & 67.17 \\
ARMADILLO2-A & 80 & 48 & 2923 & 176 & 27 & 0.18 & 31.92 \\
\hline H-PRESENT-128 [2] & 128 & 128 & 4256 & 32 & 200 & 0.18 & 110.41 \\
ARMADILLO2-B & 128 & 64 & 6025 & 64 & 1000 & 0.18 & 27.55 \\
MD4 [9] & 128 & 512 & 7350 & 456 & 112.28 & 0.13 & 20.78 \\
ARMADILLO2-B & 128 & 64 & 4353 & 256 & 250 & 0.18 & 13.19 \\
MD5 [9] & 128 & 512 & 8400 & 612 & 83.66 & 0.13 & 11.86 \\
\hline ARMADILLO2-C & 160 & 80 & 7492 & 80 & 100 & 0.18 & 17.81 \\
ARMADILLO2-C & 160 & 80 & 5406 & 320 & 250 & 0.18 & 8.55 \\
SHA-1 [9] & 160 & 512 & 8120 & 1274 & 40.18 & 0.35 & 6.10 \\
\hline ARMADILLO2-D & 192 & 96 & 8999 & 96 & 100 & 0.18 & 12.35 \\
C-PRESENT-192 [2] & 192 & 192 & 8048 & 108 & 59.26 & 0.18 & 9.15 \\
ARMADILLO2-D & 192 & 96 & 6554 & 384 & 25 & 0.18 & 5.82 \\
\hline MAME [24] & 256 & 256 & 8100 & 96 & 266.67 & 0.18 & 40.64 \\
ARMADILLO2-E & 256 & 128 & 11914 & 128 & 100 & 0.18 & 7.05 \\
SHA-256 [9] & 256 & 512 & 10868 & 1128 & 45.39 & 0.35 & 3.84 \\
ARMADILLO2-E & 256 & 128 & 8653 & 512 & 25 & 0.18 & 3.34 \\
\hline
\end{tabular}


Table 4. Implementation comparison for encryption with throughput at $100 \mathrm{kHz}$.

\begin{tabular}{cccccccc}
\hline Algorithm & $\begin{array}{c}\text { Key } \\
\text { (bits) }\end{array}$ & $\begin{array}{c}\text { Block } \\
\text { (bits) }\end{array}$ & $\begin{array}{c}\text { Area } \\
(\mathrm{GE})\end{array}$ & $\begin{array}{c}\text { Time } \\
\text { (cycles/block) }\end{array}$ & $\begin{array}{c}\text { Throughput } \\
(\mathrm{kb} / \mathrm{s})\end{array}$ & $\begin{array}{c}\text { Logic } \\
(\mu \mathrm{m})\end{array}$ & $\begin{array}{c}\text { FOM } \\
\text { (nanobit/cycle.GE }{ }^{2} \text { ) }\end{array}$ \\
\hline \hline DES [18] & 56 & 64 & 2309 & 144 & 44 & 0.18 & 83.36 \\
\hline PRESENT-80 [1] & 80 & 64 & 1570 & 32 & 200 & 0.18 & 811.39 \\
Grain [11] & 80 & 1 & 1294 & 1 & 100 & 0.13 & 597.22 \\
KTANTAN64 [3] & 80 & 64 & 927 & 128 & 50 & 0.13 & 581.85 \\
KATAN64 [3] & 80 & 64 & 1269 & 85 & 75 & 0.13 & 467.56 \\
ARMADILLO2-A & 80 & 128 & 4030 & 44 & 291 & 0.18 & 179.12 \\
Trivium [11] & 80 & 1 & 2599 & 1 & 100 & 0.13 & 148.04 \\
PRESENT-80 [19] & 80 & 64 & 1075 & 563 & 11 & 0.18 & 98.37 \\
ARMADILLO2-A & 80 & 128 & 2923 & 176 & 73 & 0.18 & 85.12 \\
\hline mCrypton [14] & 96 & 64 & 2681 & 13 & 500 & 0.13 & 684.96 \\
PRESENT-128 [1] & 128 & 64 & 1886 & 32 & 200 & 0.18 & 562.27 \\
HIGHT [13] & 128 & 64 & 3048 & 34 & 189 & 0.25 & 202.61 \\
TEA [23] & 128 & 64 & 2355 & 64 & 100 & 0.18 & 180.31 \\
ARMADILLO2-B & 128 & 192 & 6025 & 64 & 300 & 0.18 & 82.64 \\
ARMADILLO2-B & 128 & 192 & 4353 & 256 & 75 & 0.18 & 39.58 \\
AES-128 [8] & 128 & 128 & 3400 & 1032 & 12 & 0.35 & 10.73 \\
\hline ARMADILLO2-C & 160 & 240 & 7492 & 80 & 300 & 0.18 & 53.45 \\
ARMADILLO2-C & 160 & 240 & 5406 & 320 & 75 & 0.18 & 25.66 \\
\hline DESXL [18] & 184 & 64 & 2168 & 144 & 44 & 0.18 & 94.56 \\
\hline ARMADILLO2-D & 192 & 288 & 8999 & 96 & 300 & 0.18 & 37.04 \\
ARMADILLO2-D & 192 & 288 & 6554 & 384 & 75 & 0.18 & 17.46 \\
\hline ARMADILLO2-E & 256 & 384 & 11914 & 128 & 300 & 0.18 & 21.13 \\
ARMADILLO2-E & 256 & 384 & 8653 & 512 & 75 & 0.18 & 10.02 \\
\hline
\end{tabular}

\section{Conclusions}

This paper suggested a new hardware dedicated cryptographic function design called ARMADILLO. Applications for ARMADILLO include MACs, hashing for challengeresponse protocols, PRNG and as a stream cipher.

\section{References}

1. Bogdanov,A., Knudsen,L.R., Leander,G., Paar,C., Poschmann,A., Robshaw,M.J.B., Seurin,Y., Vikkelsoe,C.: Present: a Ultra-Lightweight Block Cipher. CHES'07, LNCS, vol. 4727, pp. 450-466. Springer (2007)

2. Bogdanov,A., Leander,G., Paar,C., Poschmann,A., Robshaw,M.J.B., Seurin,Y.: Hash Functions and RFID Tags: Mind the Gap. CHES'08, LNCS, vol. 5154, pp. 283-299. Springer (2008)

3. De Cannière,C., Dunkelman,O., Knežević,M.: KATAN \& KTANTAN: a Family of Small and Efficient Hardware-Oriented Block Ciphers. CHES'09, LNCS, vol. 5747, pp. 272-288, Springer (2009)

4. De Cannière,C., Preneel,B.: Trivium Specifications. eSTREAM technical report (2006) http://www.ecrypt.eu.org/stream/ciphers/trivium/trivium.pdf

5. Daemen,J., Govaerts,R., Vandewalle,J.: A Hardware Design Model for Cryptographic Algorithms. ESORICS'92, LNCS, vol. 648, pp. 419-434. Springer (1992)

6. Daemen,J., Govaerts,R., Vandewalle,J.: A Framework for the Design of One-Way Hash Functions Including Cryptanalysis of Damgård One-way Function based on a Cellular Automaton. ASIACRYPT'91, LNCS, vol. 739, pp. 82-96. Springer (1991)

7. Damgård, I.B.: A Design Principle for Hash Functions. CRYPTO'89, LNCS, vol. 435, pp. 416-427. Springer (1989) 
8. Feldhofer,M., Dominikus,S., Wolkerstorfer,J.: Strong Authentication for RFID Systems Using the AES Algorithm. CHES'04, LNCS, vol. 3156, pp. 357-370, (2004)

9. Feldhofer,M., Rechberger,C.: A Case Against Currently Used Hash Functions in RFID Protocols. On the Move to Meaningful Internet Systems OTM'06, LNCS, vol. 4277, pp. 372 381. Springer (2006)

10. Garber,D.: Braid Group Cryptography. CoRR, vol. abs/0711.3941, pp. 1-75 (2007)

11. Good,T., Chelton,W., Benaissa,M.: Hardware Results for Selected Stream Cipher Candidates. Presented at the State of the Art of Stream Ciphers SASC'07, Bochum, Germany (2007)

12. Hell,M., Johansson,T., Meier,W.: Grain: a Stream Cipher for Constrained Environments. International Journal of Wireless and Mobile Computing. vol. 2, pp. 86-93 (2007)

13. Hong,D., Sung,J., Hong,S., Lim,J., Lee,S., Koo,B.S., Lee,C., Chang,D., Lee,J., Jeong,K., Kim,H., Kim,J., Chee,S.: HIGHT: a New Block Cipher suitable for Low-Resource Device. CHES'06, LNCS, vol. 4249, pp. 46-59. Springer (2006)

14. Lim,C., Korkishko,T.: mCrypton: A Lightweight Block Cipher for Security of Lowcost RFID Tags and Sensors. Information Security Applications WISA'05, LNCS, vol. 3786, pp. 243258. Springer (2005)

15. Merkle,R.C.: One way Hash Functions and DES. CRYPTO'89, LNCS, vol. 435, pp. 416427. Springer (1989)

16. Moldovyan,A.A., Moldovyan,N.A.: A cipher based on data-dependent permutations. Journal of Cryptology, (15):1, pp. 61-72 (2002)

17. Ouafi,K., Vaudenay,S.: Pathchecker: An RFID Application for Tracing Products in SupplyChains. Presented at the International Conference on RFID Security 2009, Leuven, Belgium (2009)

18. Poschmann,A., Leander,G., Schramm,K., Paar,C.: New Lightweight DES Variants Suited for RFID Applications. FSE'07, LNCS, vol. 4593, pp. 196-210. Springer (2007)

19. Rolfes,C., Poschmann,A., Leander,G., Paar,C.: Ultra-Lightweight Implementations for Smart Devices - Security for 1000 Gate Equivalents. CARDIS 2008, LNCS, vol. 5189, pp. 89-103. Springer (2008)

20. Secure Hash Standard. Federal Information Processing Standard publication \#180-2. U.S. Department of Commerce, National Institute of Standards and Technology (2002)

21. Tillich,J.P., Zémor,G.: Hashing with SL 2 . CRYPTO'94, LNCS, vol. 839, pp. 40-49. Springer (1994)

22. Wheeler,D.J., Needham,R.M.: TEA: a Tiny Encryption Algorithm. FSE'94, LNCS, vol. 809, pp. 363-366. Springer (1994)

23. Yu,Y., Yang,Y., Fan,Y., Min,H.: Security Scheme for RFID Tag. Technical report WPHARDWARE-022, Auto-ID Labs white paper (2006) http://www.autoidlabs.org/singleview/dir/article/6/230/page.html

24. Yoshida,H., Watanabe,D., Okeya,K., Kitahara,J., Wu,J., Küçük,Ö., Preneel,B.: MAME: A Compression Function With Reduced Hardware Requirements. CHES'07, LNCS, vol. 4727, pp. 148-165. Springer (2007) 